\title{
Characterization of AmpC, CTX-M and MBLs Types of $\beta$-lactamases in Clinical Isolates of Klebsiella pneumoniae and Escherichia coli Producing Extended Spectrum $\beta$-lactamases in Kerman, Iran
}

\author{
Shahla Mansouri ${ }^{1}$; Davood Kalantar Neyestanaki ${ }^{2,{ }^{*}}$; Mostafa Shokoohi ${ }^{3}$; Shahnaz Halimi ${ }^{2}$; \\ Reza Beigverdi ${ }^{2}$; Fereshteh Rezagholezadeh ${ }^{2}$; Ali Hashemi ${ }^{4}$ \\ ${ }^{1}$ Department of Microbiology, School of Medicine, Kerman University of Medical Sciences, Kerman, IR Iran \\ ${ }_{3}^{2}$ Department of Microbiology, School of Medicine, Tehran University of Medical Sciences. Tehran, IR Iran \\ ${ }_{4}^{3}$ Physiology Research Center, Kerman University of Medical Sciences, Kerman, IR Iran \\ ${ }^{4}$ Department of Microbiology, School of Medicine, Shahid Beheshti University of Medical Sciences. Tehran, IR Iran \\ ${ }^{*}$ Corresponding author: Davood Kalantar Neyestanaki, Department of Microbiology, School of Medicine, Tehran University of Medical Sciences, Tehran, IR Iran. Tel/Fax:+98- \\ 2188955810, E-mail: davoud1362@gmail.com
}

Received: October 24, 2012; Revised: January 23, 2013; Accepted: February 19, 2013

\begin{abstract}
Background: Extended spectrum $\beta$-lactamases (ESBLs) and AmpC $\beta$-lactamases enzyme are major sources of resistance to $\beta$-lactam antibiotics especially in Enterobacteriaceae such as Escherichia coli and Klebsiella pneumoniae. Increasing frequency of the co-existence of ESBLs with AmpC- $\beta$-lactamases in bacteria is a serious threat for treating bacterial infections.

Objectives: The aim of this study was to determine the presence of AmpC and CTX-M types of $\beta$-lactamases in clinical isolates of $E$. coli and K. pneumoniae producing ESBLs.

Materials and Methods: Resistance to different antibiotics was determined using the standard disk diffusion method. ESBLs, MBLs and AmpC- $\beta$-lactamases were detected by the combination double disk test (CDDT) method and polymerase chain reaction (PCR) was used to determine $b l a_{C T X-M}$ genes in the ESBLs and AmpC positive isolates.

Results: The prevalence of ESBLs and AmpC- $\beta$-lactamase producer isolates was 181 (43.8\%) and 133 (37.2\%), respectively. The prevalence of bla $_{\text {CTX-M }}$ among isolates was $61(14.7 \%)$.

Conclusions: Outbreak of isolates co-expressing AmpC- $\beta$-lactamases and ESBLs can cause serious problems in the future, regarding the treatment of infections caused by these common enteric pathogens.
\end{abstract}

Keywords: Antibiotic Resistance; ESBLs; AmpC- $\beta$-lactamases; bla ${ }_{\text {CTX-M }}$ Escherichia coli; Klebsiella pneumoniae

\section{Background}

Escherichia coli and Klebsiella pneumoniae are important causes of different bacterial infections, including cholecystitis, bacteremia, cholangitis, urinary tract infections (UTI), neonatal meningitis and pneumonia $(1,2)$. The $\beta$-lactams antibiotics are one of the treatment choices for these bacterial infections (3). One of the main mechanisms of resistance to $\beta$-lactams antibiotics is via the actions of $\beta$-lactamase enzymes (1). Extended spectrum $\beta$-lactamases (ESBLs) are $\beta$-lactamases that hydrolyze extended-spectrum cephalosporins such as cefotaxime and ceftazidime(4). Metallo- $\beta$-lactamases (MBLs) are a diverse group of $\beta$-lactamases that are active not only against the cephalosporins but also against the carbapenems (5). According to Ambler classification, AmpC- $\beta$-lactamases are an important group of class $C \beta$-lactamases that hydrolize penicillins, extended-spectrum cephalosporins, cephamycins and aztreonam, however they can't be in- hibited by $\beta$-lactamase inhibitors such as clavulanate, sulbactam and tazobactam, but are inhibited by phenylboronic acid and cloxacillin $(6,7)$.

ESBLs and AmpC $\beta$-lactamases may co-exist, thus their detection is difficult because they mask each other and cause an increase in the minimum inhibitory concentrations for $\beta$-lactamas antibiotics (7). CTX-M type of the $\beta$-lactamase enzymes is a ESBLs type that is widely reported in Enterobacteriaceae such as E. coli and K. pneumoniae. This enzyme is the predominant ESBLs type in some countries (8). The CTX-M enzymes are usually encoded by genes that are carried on the plasmid and have greater activity against cefotaxime than other oxyimino-cephalosporins such as ceftazidime (9).

\section{Objectives}

The aim of this study was to determine the presence and prevalence of MBLs, AmpC and CTX-M types of

Implication for health policy/practice/research/medical education:

This study can help physicians choose better antibiotics for treatment the Entrobacteriacea infections.

Copyright @ 2014, Ahvaz Jundishapur University of Medical Sciences; Published by Kowsar Corp. This is an open-access article distributed under the terms of the Creative Commons Attribution License, which permits unrestricted use, distribution, and reproduction in any medium, provided the original work is properly cited. 
$\beta$-lactamases among clinical isolates of E. coli and K. pneumoniae producing ESBLs in Kerman, Iran.

\section{Materials and Methods}

\subsection{Bacterial Strains}

In total, 413 consecutive non-duplicate $E$. coli $(\mathrm{n}=338)$ and $K$. pneumoniae $(\mathrm{n}=75)$ were isolated from clinical specimens including blood, urine and body fluids of patients admitted to three major hospitals (Afzali Poor, Kashani and Bahonar) located in three different regions of Kerman, southeast Iran from November 2007 to July 2008. The isolates were identified by their cultural characteristics and reactions to standard biochemical tests.

\subsection{Antimicrobial Susceptibility Testing}

The antibiotic resistance pattern of isolates was determined by using the disk diffusion method according to the Clinical and Laboratory Standard Institute (CLSI) guidelines (10). The antibiotics tested were ceftizoxime $(30 \mu \mathrm{g})$, cefotaxime $(30 \mu \mathrm{g})$, ceftazidime $(30 \mu \mathrm{g})$, cephalexin $(30 \mu \mathrm{g})$, amoxicillin $(30 \mu \mathrm{g})$, imipenem $(10 \mu \mathrm{g})$, cefepime $(30 \mu \mathrm{g})$, cefoxitin $(30 \mu \mathrm{g})$, gentamycin $(30 \mu \mathrm{g})$, tetracycline (30 $\mu \mathrm{g})$, trimethoprim/sulfamethoxazole (30 $\mu \mathrm{g})$, nalidixic acid and ciprofloxacin $(30 \mu \mathrm{g})$ (Himedia, India). E. coli ATCC 25922 and K. pneumoniae 700603 were used as quality control strains for antimicrobial susceptibility testing.

\subsection{Detection of ESBLs Producing Isolates}

ESBLs producing isolates were detected using the combination double disk test (CDDT) as a standard disk diffusion assay on Mueller Hinton agar (Himedia, India) (11). ESBLs presence was assayed using the following antibiotic disks: ceftazidime (CAZ) $(30 \mu \mathrm{g})$, ceftazidime (30 $\mu \mathrm{g})$ plus clavulanic acid (CA) (10 $\mu \mathrm{g})$, cefotaxime (CTX) (30 $\mu \mathrm{g})$, cefotaxime $(30 \mu \mathrm{g})$ plus clavulanic acid $(10 \mu \mathrm{g})$, and cefpodoxime $(30 \mu \mathrm{g})$, cefpodoxime $(30 \mu \mathrm{g})$ plus clavulanic acid $(10 \mu \mathrm{g})$ (MAST Chemical Co, England). The disk with CA and without CA was placed on the inoculated surface of the Mueller-Hinton agar (Himedia, India) plate by the standard disk diffusion method. The plates were then incubated overnight at $37^{\circ} \mathrm{C}$ in ambient air. An increase of $\geq 5 \mathrm{~mm}$ in zone diameter of CAZ, CPD and/or CTX tested in combination with CA (CAZ-CA, CPD-CA and/or CTXCA) versus CAZ, CPD and/or CTX alone was considered positive for ESBLs. E. coli ATCC 25922 and K. pneumoniae 700603 were used as control strains for detection of ESBLs producing isolates.

\subsection{Determination of AmpC Producing Isolates}

AmpC phenotype was specified by means of compound disk using cefoxitin (FOX), cefepime (CPM), CTX and CAZ disks $(30 \mu \mathrm{g})$ alone and in combination with $400 \mu \mathrm{g}$ of phenylboronic acid (BA) (SIGMA-ALDRICH, Fluka, China). The disk with BA and without BA was placed on the inoculated surface of the Mueller-Hinton agar plate (Himedia, India) by the standard disk diffusion method. The plates were then incubated overnight at $37^{\circ} \mathrm{C}$ in ambient air. An increase of $\geq 5 \mathrm{~mm}$ in zone diameter of CAZ, CTX, FOX and/or CPM tested in combination with BA (CAZ, CTX, FOX-BA and/or CPM-BA) versus CAZ, CTX, FOX and/or CPM alone was considered positive for AmpC $\beta$-lactamases (12).

\subsection{Determination of MBLs Producing Isolates}

Disks containing imipenem and imipenem with $5 \mu \mathrm{L}$ EDTA (0.5 M) (SIGMA-ALDRICH, China) were used for determination of the presence of MBLs. An increase of $\geq$ $7 \mathrm{~mm}$ in zone diameter of imipenem tested in combination with EDTA versus imipenem alone was considered as MBLs positive (13).

\subsection{DNA Extraction and Amplification}

Total DNA template was extracted as described previously (13). The primers used for PCR amplification were bla $_{\text {CTX-M }}$ F-5'- CGC TTT GCG ATG TGA AG-3' and bla $a_{\text {CTX-M }}$ R-5'ACC GCG ATA TCG TTG GT-3' (14). The PCR reactions were carried out in a Primus thermo cycler by using the PCR Master Kit (Cinna Clone Inc., Iran) according to the manufacturer guideline. PCR condition was as follows: initial denaturation at $95^{\circ} \mathrm{C}$ for 4 minutes followed by 30 cycles of denaturation at $95^{\circ} \mathrm{C}$ for 1 minute, annealing for $1 \mathrm{~min}$ ute at $60^{\circ} \mathrm{C}$, extension at $72^{\circ} \mathrm{C}$ for 1 minute. The final extension step was continued for another 5 minutes at $72^{\circ} \mathrm{C}$.

\subsection{Statistical Analysis}

Statistical analysis was carried out using the SPSS 15 statistical software. We used the Chi-squared analysis for comparison of data.

\section{Results}

A total of 338 strains of $E$. $\operatorname{coli}$ (urine $=316$, blood $=14$, other body fluids $=8$ ) and 75 strains of $K$. pneumoniae (urine $=57$, blood $=13$, other body fluids $=5$ ) were collected from three hospitals in Kerman, Iran. The rate of resistance to different antibiotics in K. pneumoniae and E. coli are shown in Table 1.

In this study, only two K. pneumoniae were resistant to imipenem, which were isolated from blood. Eighty-four percent of the isolates of K. pneumoniae were resistant to at least one antibiotic and 12 (16\%) isolates were susceptible to all tested antibiotics.

More than 50 \% of K. pneumoniae isolates were resistant to amoxicillin, cephalexin, ceftazidime and gentamycin. Among 75 K. pneumoniae isolates 33 (44\%) produced ESBLs, 21 (28\%) produced AmpC $\beta$-lactamases and 1 (1.3\%) produced MBLs (Figures 1 and 2). 
Mansouri S et al.

Table 1. Rate of resistance to different antibiotics

\begin{tabular}{|c|c|c|}
\hline Antibiotics & E. coli No. (\%) & K. pneumoniae, No. (\%) \\
\hline IMP $^{\mathrm{a}}$ & $0(0)$ & $2(2.6)$ \\
\hline $\mathbf{C T}^{\mathrm{a}}$ & $43(12.7)$ & $18(24)$ \\
\hline CTX $^{\mathrm{a}}$ & $105(31)$ & $25(33)$ \\
\hline $\mathbf{G M}^{\mathrm{a}}$ & $133(39.3)$ & $48(64)$ \\
\hline $\mathbf{C P M}^{\mathrm{a}}$ & $139(41.1)$ & $27(36)$ \\
\hline $\mathbf{C P}^{\mathrm{a}}$ & $152(44.9)$ & $21(28)$ \\
\hline FOX $^{\mathrm{a}}$ & $156(46.1)$ & $29(38)$ \\
\hline $\mathbf{C A Z}^{\mathrm{a}}$ & $188(55.6)$ & $52(69)$ \\
\hline $\mathbf{C N}^{\mathrm{a}}$ & $213(63)$ & $54(72)$ \\
\hline $\mathbf{N A}^{\mathrm{a}}$ & $243(71.9)$ & $30(40)$ \\
\hline TET $^{\mathrm{a}}$ & $283(83.7)$ & $40(53)$ \\
\hline $\mathbf{A M X}^{\mathrm{a}}$ & $309(91.4)$ & $63(84)$ \\
\hline SXT $^{\mathrm{a}}$ & $316(93.4)$ & $35(47)$ \\
\hline
\end{tabular}

Figure 1. Extended spectrum $\beta$-Lactamases producing K. pneumoniae

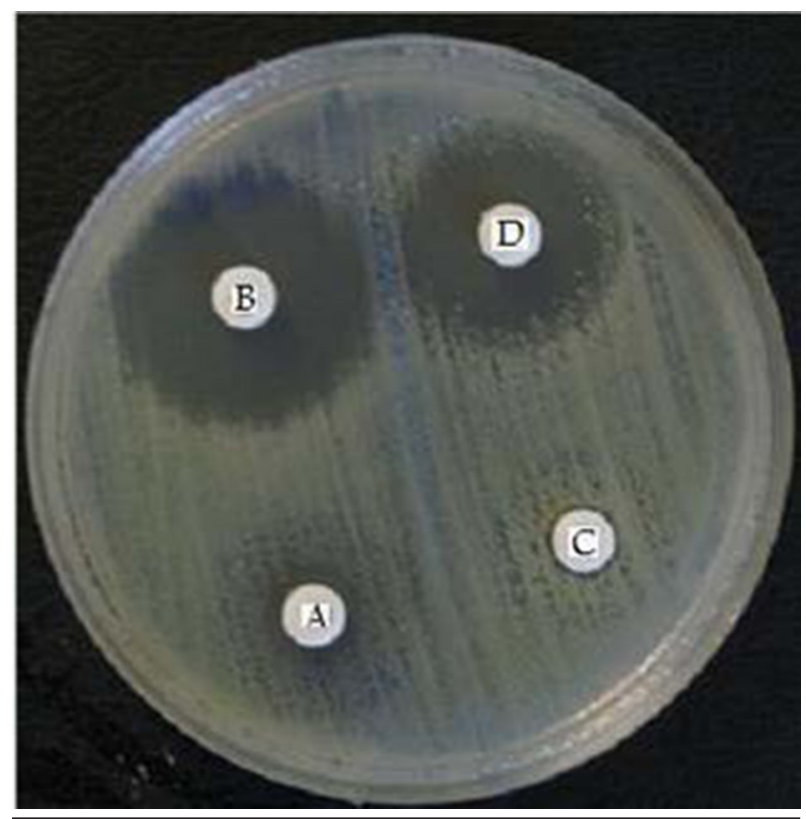

( $\mathrm{A}=$ Cefotaxime $\mathrm{B}=$ Cefotaxime + Clavulanic acid $) \mathrm{C}=$ Ceftazidime $\mathrm{D}=$ Ceftazidime + Clavulanic acid) Clavulanic acid inhibited an extended spectrum of $\beta$-lactamases with the occurrence of a growth inhibition zone.
Figure 2. MBLs Producing K. pneumoniae

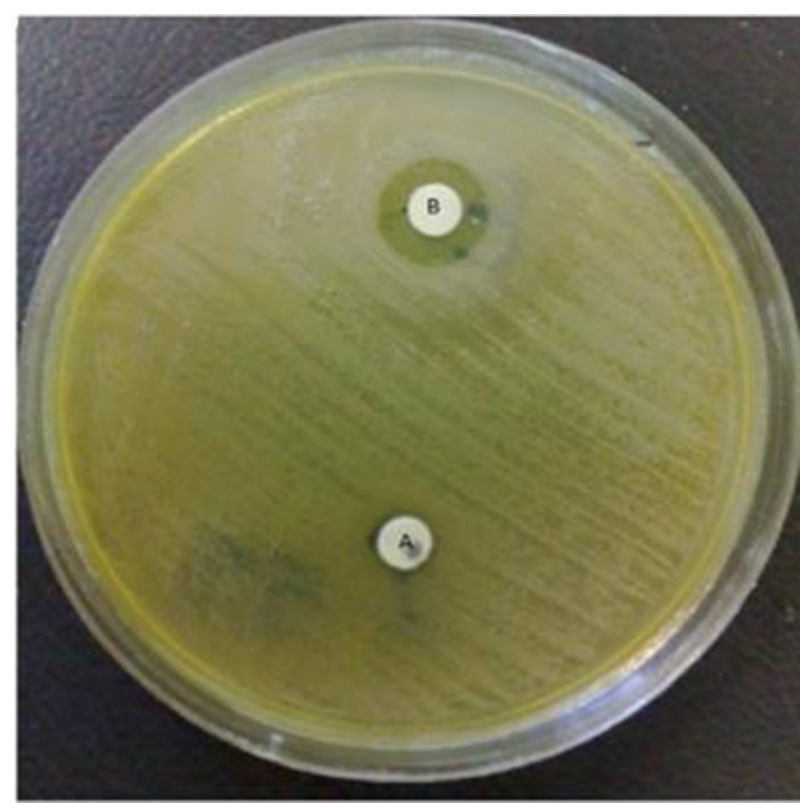

$\mathrm{A}=$ Imipenem, $\mathrm{B}=$ Imipenem +EDTA (.0.5 M).

Simultaneous production of ESBLs and AmpC $\beta$-lactamases were observed in $21(28 \%)$ isolates of $K$. pneumoniae (Table 2). More than 321 (95\%) of the isolates of $E$. coli were resistant to at least one of the following antibiotics, amoxicillin, cephalexin, tetracycline, trimethoprim/sulfamethoxazole, nalidixic acid, ciprofloxacin and gentamicin. Out of the $338 \mathrm{E}$. coli isolates, 148 (43.7\%) produced ESBLs and 133 (39.3\%) produced AmpC $\beta$-lactamases. Six isolates of E. coli only produced AmpC $\beta$-lactamases. Co-existence of ESBLs and AmpC $\beta$-lactamases were observed in 133(39.3\%) isolates (Table 3).

All ESBLs and AmpC producer isolates were multi drug resistant and showed resistance to four different antibiotics (Tables 2 and 3).

None of the AmpC $\beta$-lactamases producing isolates were resistant to imipenem. From a total of $133 \mathrm{AmpC}$ positive isolates, 19 were detected with cefoxitin, 81 were detected with cefepime, 82 were detected with cefotaxime and 91 isolates were detected with ceftazidime. The prevalence rate of bla $a_{\mathrm{CTX}-\mathrm{M}}$ gene among ESBLs and AmpC producing isolates of K. pneumoniae and E. coli were 16 (21.3\%) and 45 (13.3\%) respectively (Figure 3). 
Mansouri S et al.

\begin{tabular}{|c|c|c|c|c|c|}
\hline \multirow[t]{2}{*}{ Antibiotic Resistance Patterns ${ }^{a}$} & \multicolumn{4}{|c|}{ Isolates No. } & \multirow[b]{2}{*}{ Others } \\
\hline & Blood & Urine & AmpC & blaCTX-M & \\
\hline CP, CTX, CAZ, CT, AMX, GM, NA, TET, CN, SXT, FOX, CPM & - & 3 & 3 & 2 & - \\
\hline CTX, CAZ, CT, AMX, GM, NA, TET, CN, SXT, FOX, CPM & 1 & 1 & 1 & 1 & - \\
\hline CP, CAZ, CT, AMX, GM, NA, TET, CN, SXT, FOX, CPM & - & 2 & 2 & - & - \\
\hline CP, CTX, CAZ, CT, AMX, GM, NA, TET, CN, SXT, FOX & - & 2 & 2 & - & - \\
\hline CTX, CAZ, AMX, GM, NA, TET, CN, SXT, FOX, CPM & - & 1 & 1 & 1 & - \\
\hline CP, CTX, CAZ, AMX, GM, TET, CN, SXT, FOX, CPM & - & 1 & - & - & - \\
\hline CP, CAZ, CT, AMX, GM, NA, TET, CN, SXT, FOX & - & 1 & - & - & - \\
\hline CP, CTX, CAZ, AMX, TET, CN, SXT, FOX, CPM & - & 1 & 1 & - & - \\
\hline CTX, CAZ, AMX, GM, NA, CN, SXT, FOX, CPM & - & - & - & - & 1 \\
\hline CTX, CAZ, AMX, GM, CN, SXT, FOX, CPM & 6 & 2 & 9 & 8 & 1 \\
\hline CTX, CAZ, AMX, NA, CN, SXT, FOX, CPM & - & 1 & 1 & 1 & - \\
\hline IMP, CTX, CT, AMX, GM, CN, FOX, CPM & 1 & - & - & 1 & - \\
\hline CAZ, AMX, GM, TET, CN, SXT, FOX, CPM & 1 & - & - & - & - \\
\hline CTX, CAZ, AMX, GM, CN, FOX, CPM & - & 1 & - & 1 & - \\
\hline CAZ, AMX, GM, CN, SXT, FOX, CPM & - & 1 & 1 & - & - \\
\hline CTX, AMX, GM, CN, SXT, FOX, CPM & 1 & - & - & - & - \\
\hline CTX, CAZ, AMX, CN, FOX, CPM & - & 1 & - & - & - \\
\hline CTX, CAZ, CN, SXT, FOX, CPM & - & 1 & - & - & - \\
\hline IMP, CT, AMX, GM, TET, CN & 1 & - & - & - & - \\
\hline CTX, CAZ, AMX, CN & - & 1 & - & 1 & - \\
\hline
\end{tabular}

${ }^{\mathrm{a}}$ For abbreviations please refer to Table 1.

Table 3. Antibiotic resistance patterns of E.coli isolates producing ESBLs

\begin{tabular}{|c|c|c|c|c|c|}
\hline \multirow[b]{2}{*}{ Antibiotic Resistance Patterns ${ }^{a}$} & \multicolumn{4}{|c|}{ Isolates No. } & \multirow[b]{2}{*}{ Other } \\
\hline & Blood & Urines & AmpC & blaCTX-M & \\
\hline CP, CAZ, CT, AMX,GM, NA,TET, CN, SXT, FOX, CPM, CTX & 1 & 10 & 11 & 6 & - \\
\hline CAZ, CT, AMX, GM, NA, TET, CN, SXT, FOX, CPM, CTX & - & 2 & - & 1 & - \\
\hline CP, CAZ, CT, AMX, NA,TET, CN, SXT, FOX, CPM, CTX & 1 & 4 & 4 & 2 & - \\
\hline CP, CAZ, CT, AMX,GM, NA, CN, SXT, FOX, CPM, CTX & - & - & 1 & - & 1 \\
\hline CP, CAZ, AMX,GM, NA,TET, CN, SXT, FOX, CPM, CTX & 4 & 16 & 16 & 6 & - \\
\hline CP, CAZ, CT, AMX, GM, NA,TET, CN, SXT, FOX, CPM & - & 1 & 2 & - & 1 \\
\hline CAZ, CT, AMX, GM, TET, CN, SXT, FOX, CPM, CTX & - & 1 & 1 & 1 & - \\
\hline CAZ, AMX, GM, NA, TET, CN, SXT, FOX, CPM, CTX & - & 2 & 3 & 2 & 1 \\
\hline CAZ, CT, AMX, NA, TET, CN, SXT, FOX, CPM, CTX & - & 2 & 1 & 1 & - \\
\hline CP, CAZ, AMX, NA,TET, CN, SXT, FOX, CPM, CTX & - & 14 & 13 & 4 & - \\
\hline CP, AMX,GM, NA,TET, CN, SXT, FOX, CPM, CTX & - & 1 & 2 & 2 & 1 \\
\hline CAZ, CT, AMX, GM, NA, TET, CN, SXT, FOX,CTX & - & 1 & 1 & - & - \\
\hline CP, CAZ, AMX,GM, NA,TET, CN, SXT, FOX, CPM & - & 4 & 4 & - & - \\
\hline CAZ, AMX, GM, TET, CN, SXT, FOX, CPM, CTX & - & 1 & 1 & - & - \\
\hline CAZ, AMX, NA, TET, CN, SXT, FOX, CPM, CTX & 1 & 7 & 7 & 3 & - \\
\hline CAZ, CT, AMX, TET, CN, SXT, FOX, CPM, CTX & - & 1 & 1 & - & - \\
\hline CP, CAZ, AMX , NA, CN, SXT, FOX, CPM, CTX & - & 2 & 1 & 1 & - \\
\hline
\end{tabular}


Mansouri S et al.

CP, CAZ, AMX, TET, CN, SXT, FOX, CPM, CTX CAZ, AMX, GM, NA, TET, CN, SXT, FOX, CPM CAZ, CT, AMX, NA, TET, CN, SXT, FOX, CPM CAZ, CT, AMX, NA, CN, SXT, FOX, CPM, CTX CAZ, CT, AMX, NA, TET, CN, SXT, FOX, CPM CP, CAZ, AMX, NA,TET, CN, SXT, FOX, CTX CP, CAZ, AMX, NA,TET, CN, SXT, FOX, CPM CAZ, AMX, NA, TET, SXT, FOX, CPM, CTX CP, CAZ, AMX,GM, NA,TET, CN, SXT, FOX CAZ, AMX, TET, CN, SXT, FOX, CPM, CTX CP, CAZ,GM, NA,TET, CN, SXT, FOX, CPM CAZ, AMX, GM, CN, SXT, FOX, CPM, CTX CAZ, AMX, GM, CN, SXT, FOX, CPM, CTX CAZ, AMX, GM, TET, CN, FOX, CPM, CTX CAZ, AMX, GM, TET, CN, SXT, FOX, CPM CAZ, AMX, NA, TET, CN, SXT, FOX, CPM CAZ, AMX, GM, NA, TET, CN, SXT, FOX CP, AMX, NA,TET, SXT, FOX, CPM,CTX CP, AMX, NA,TET, CN, FOX, CPM, CTX CAZ, CT, AMX, NA, TET, CN, SXT, FOX CAZ, CT, AMX, NA,CN, SXT, FOX,CTX CP, CAZ, NA,TET, CN, SXT, FOX, CPM CAZ, AMX, GM, SXT, FOX, CPM, CTX CP, CAZ, AMX,GM, NA,TET, CN, SXT CAZ, AMX, CN, SXT, FOX, CPM, CTX CAZ, AMX, TET, CN, SXT, FOX, CPM CAZ, AMX, GM, CN, SXT, FOX, CPM CAZ, AMX, GM, CN, FOX, CPM, CTX CAZ, AMX, GM, TET, CN, SXT, FOX CAZ, AMX, NA, TET, CN, FOX, CPM CAZ, AMX, NA, TET, CN, SXT, CTX CAZ, AMX, NA, TET, CN, SXT, FOX CP, CAZ, AMX, NA, CN, SXT, FOX CAZ, AMX, TET, FOX, CPM, CTX CAZ, AMX, CN, SXT, FOX, CPM CAZ, AMX, TET, CN, SXT, CTX CAZ, AMX, GM, CN, FOX, CPM CAZ, AMX,TET, CN, FOX, CPM CAZ, AMX, GM, CN, SXT, CTX CAZ, AMX, NA, TET, CN, CTX CAZ, AMX, NA, TET, CN, SXT

\begin{tabular}{|c|c|c|c|c|}
\hline - & 1 & 1 & - & - \\
\hline - & 3 & 3 & - & - \\
\hline - & 1 & - & - & - \\
\hline - & 4 & 4 & 3 & - \\
\hline - & 1 & - & - & - \\
\hline - & 1 & - & - & - \\
\hline - & 3 & 3 & - & - \\
\hline - & 3 & 3 & 2 & - \\
\hline - & 1 & 1 & - & - \\
\hline - & 3 & 3 & - & - \\
\hline - & 1 & 1 & - & - \\
\hline - & 3 & 2 & 1 & - \\
\hline - & 1 & 1 & - & - \\
\hline - & 1 & 1 & 1 & - \\
\hline 1 & 2 & 2 & - & - \\
\hline 1 & 2 & 2 & - & - \\
\hline - & 2 & - & - & - \\
\hline - & 1 & 1 & 1 & - \\
\hline - & - & 1 & 1 & 1 \\
\hline - & 2 & 2 & - & - \\
\hline - & 1 & 1 & - & - \\
\hline - & 1 & 1 & - & - \\
\hline - & 2 & 2 & 2 & - \\
\hline - & 2 & - & - & - \\
\hline - & 2 & 2 & 1 & - \\
\hline - & 3 & 3 & - & - \\
\hline 1 & 1 & 2 & - & - \\
\hline 1 & - & 1 & - & - \\
\hline - & 1 & - & - & - \\
\hline - & 1 & 1 & - & - \\
\hline - & 1 & - & - & - \\
\hline - & 1 & 1 & - & - \\
\hline - & 1 & 1 & - & - \\
\hline - & 2 & 2 & 2 & - \\
\hline - & 1 & 1 & - & 1 \\
\hline - & 1 & - & - & - \\
\hline 1 & - & 1 & - & - \\
\hline - & 1 & 1 & - & - \\
\hline - & 1 & - & 1 & - \\
\hline - & 1 & - & 1 & - \\
\hline - & 1 & - & - & - \\
\hline
\end{tabular}

\footnotetext{
${ }^{\mathrm{a}}$ For abbreviations please refer to Table 1.
} 
Figure 3. Electrophoresis of PCR products for amplifying bla ${ }_{C T X-M}$ broad spectrum $\beta$-lactamases genes.

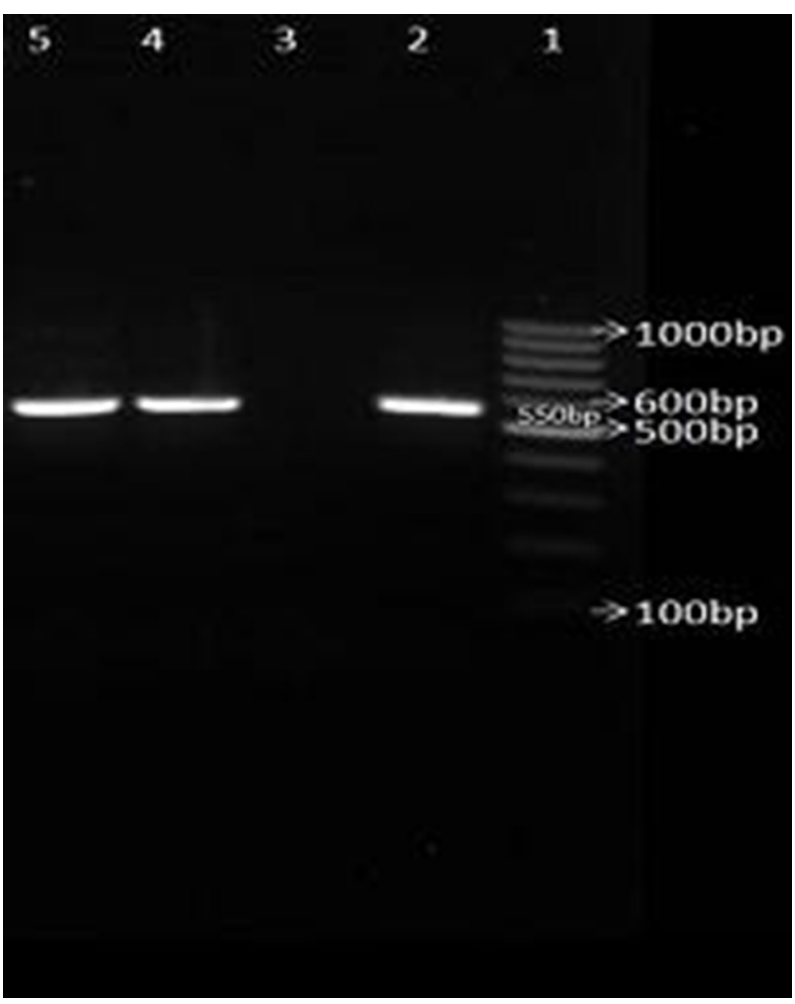

No.1: DNA marker (100 bp), No 2: positive control for bla ${ }_{C T X-M}$ No. 3: negative control No. 4,5: isolates with bla ${ }_{C T X-M}(550 \mathrm{bp})$ gene.

\section{Discussion}

Appearance of $\beta$-lactamase enzymes among Gramnegative bacteria especially those that have a key role in hospital infections, is an important concern. The prevalence of strains possessing several resistance enzymes concomitantly has caused serious problems for the treatment and the diagnosis of such strains (7). Compared to the results of previous studies in 2002, the percentages of multi-resistance isolates increased in E. coli (15). In general, ESBLs and AmpC $\beta$-lactamases producing isolates are susceptible to imipenem (4). In this study, similar to other reports, more than $99 \%$ of isolates were susceptible to imipenem. However, two isolates in our study were resistant to imipenem and one of them was a MBLs producer isolate. Fortunately, carbapenem resistance is still very rare among K. pneumoniae and $E$. coli in Iran (16-18).

In the current study, the 28 (K. pneumoniae $=6$ and $E$. coli $=22$ ) isolates of K. pneumoniae and E. coli, which were resistant to cefoxitin, ceftazidime, cefotaxim and cefepime, were not inhibited by clavulanic acid and phenylboronic acid. These results can partially be explained by the concomitant presence of several resistance mechanisms in these isolates. It may also be inferred that the procedures available for the detection of $\beta$-lactam resistance pheno- types are no longer practical. Getzlaff et al. reported that cefoxitin and cefoxitin plus phenylboronic acid had 95\% specificity for phenotypic AmpC detection but in this study only $14.2 \%$ (19/133) of AmpC producing isolates were detected by this method, which may be related to the presence of other mechanisms of resistance to cefoxitin in this area (19).

Furthermore, in our study the AmpC $\beta$-lactamases were also the source of one of the resistance mechanisms to ceftazidime, cefotaxim and cefepime. In this study, the prevalence of the phenotypes ESBLs and AmpC in E. coli was $43.7 \%$ and $39.3 \%$ and in K. pneumoniae, this was $44 \%$ and $28 \%$, respectively. The prevalence of ESBLs in E. coli and $K$. pneumoniae as reported in this study is in agreement with that of an investigation from 2007 and 2009 in Tehran (16-18). This can partially show an invariant and uniform prevalence for ESBLs across Iran

According to the literature review, the prevalence of the phenotypes of AmpC has not yet been reported for the clinical isolates of E. coli and K. pneumoniae in Iran by the use of a phenylboronic acid disk. On the other hand, the prevalence rate of AmpC has been reported for South Korea (53\%), India (47.3\%) and Singapore (57\%), which are higher than the prevalence rate in $\operatorname{Iran}(20,21)$. There was a significant correlation between presence of bla $a_{\text {CTX-M }}$ and resistance to cefotaxime and ceftizoxime in our isolates $(\mathrm{P}<0.05)$. CTX-M producing isolates amongst $E$. coli and $K$. pneumoniae have been reported from other parts of Iran such as Tehran and Kurdistan $(16,22,23)$. Also, CTX$\mathrm{M}$ producing isolates have also been identified in Korea, Italy, France, New Zealand, Egypt and other countries and carriers of CTX-M type of $\beta$-lactamases were subsequently hospitalized (21,24-27). This enzyme has now become the most prevalent $\beta$-lactamases in hospitals and in the community (16).

The emergence of the co-production of ESBLs, AmpC and MBLs is increasing in Iran. The prevalence of isolates possessing several resistance enzymes concomitantly has caused serious problems for treatment and diagnosis. Consequently, regular epidemiological assessments on the drug resistance patterns of the isolates responsible for nosocomial infections and determination of the molecular resistance mechanisms can be useful for the empirical treatment of the infections and prevention of the drug resistance distribution.

\section{Acknowledgements}

None declared.

\section{Authors' Contribution}

All authors were involved in: Study design, data collection, article approval, statistical analysis

\section{Financial Disclosure}

There is no financial disclosure. 


\section{Funding/Support}

This research was supported by grant No. 88/02 from the Research Council of Kerman University of Medical Sciences.

\section{References}

1. Kalantar Davoud, Mansouri Shahla. Emergence of multiple $\beta$-lactamases produced by Escherichia coli clinical isolates from hospitalized patient in Kerman, Iran. Jundishapur J Microbiol. 2011;3(4):137-145.

2. Paterson DL. Resistance in gram-negative bacteria: enterobacteriaceae. Am J Med. 2006;119(6 Suppl 1):S20-8.

3. Perez F, Endimiani A, Hujer KM, Bonomo RA. The continuing challenge of ESBLs. Curr Opin Pharma col. 2007;7(5):459-69.

4. Poole K. Pseudomonas aeruginosa: resistance to the max. Front Microbiol. 2011;2:65.

5. Kalantar D, Mansouri Sh, Razavi M. Emergence of imipenem resistance and presence of metallo- $\beta$-lactamases enzymes in multi drug resistant Gram negative bacilli isolated from clinical samples in Kerman, 2007-2008. J Kerman Univ Med Sci. 2010;17:208-14.

6. Shahid Mohammad, Sobia Farrukh, Singh Anuradha, Khan Haris M, Hawkey Peter M, Huq Anwar, et al. AmpC $\beta$-lactamases and bacterial resistance: an updated mini review. Rev Med Microbiol. 2009;20(3):41-55.

7. Sundin Daniel R. Hidden Beta-Lactamases in the Enterobacteriaceae - Dropping the Extra Disks for Detection, Part II. Clin Microbiol Newsletter. 2009;31(7):47-52.

8. Coque TM, Baquero $\mathrm{F}$, Canton R. Increasing prevalence of ESBL-producing Enterobacteriaceae in Europe. Euro Surveill. 2008;13(47).

9. Bush K, Jacoby GA. Updated functional classification of beta-lactamases. Antimicrob Agents Chemother. 2010;54(3):969-76.

10. Cockerill Franklin R. Performance Standards for Antimicrobial Susceptibility Testing: Twenty-first Informational Supplement.: Clinical and Laboratory Standards Institute (CLSI); 2011.

11. Paterson DL, Bonomo RA. Extended-spectrum beta-lactamases: a clinical update. Clin Microbiol Rev. 2005;18(4):657-86.

12. Song W, Jeong SH, Kim JS, Kim HS, Shin DH, Roh KH, et al. Use of boronic acid disk methods to detect the combined expression of plasmid-mediated AmpC beta-lactamases and extendedspectrum beta-lactamases in clinical isolates of Klebsiella spp., Salmonella spp., and Proteus mirabilis. Diagn Microbiol Infect Dis. 2007;57(3):315-8.

13. Pitout JD, Gregson DB, Poirel L, McClure JA, Le P, Church DL. Detection of Pseudomonas aeruginosa producing metallo-betalactamases in a large centralized laboratory. J Clin Microbiol. 2005;43(7):3129-35.

14. Brasme L, Nordmann P, Fidel F, Lartigue MF, Bajolet O, Poirel L, et al. Incidence of class A extended-spectrum beta-lactamases in Champagne-Ardenne (France): a 1 year prospective study. J Antimicrob Chemother. 2007;60(5):956-64.
15. Mansouri S, Shareifi S. Antimicrobial resistance pattern of Escherichia coli causing urinary tract infections, and that of human fecal flora, in the southeast of Iran. Microb Drug Resist. 2002;8(2):123-8

16. Feizabadi MM, Delfani S, Raji N, Majnooni A, Aligholi M. Distribution of blaTEM, blaSHV, blaCTX-M Genes Among Clinical Isolates of Klebsiella pneumoniae at Labbafinejad Hospital, Tehran, Iran. Microbiol Drug Resistance. 2009:1-5.

17. Shahcheraghi F, Moezi H, Feizabadi MM. Distribution of TEM and SHV beta-lactamase genes among Klebsiella pneumoniae strains isolated from patients in Tehran. Med Sci Monit. 2007;13(11):BR247-250.

18. Shahcheraghi Fereshteh, Nasiri Siavash, Noveiri Hanieh. The Survey of Genes Encoding Beta-Lactamases, in Escherichia Coli Resistant to Beta-Lactam and Non-Beta-Lactam Antibiotics. Iran JBasic Med. 2010;13(1):230-237.

19. Peter-Getzlaff S, Polsfuss S, Poledica M, Hombach M, Giger J, Bottger EC, et al. Detection of AmpC beta-lactamase in Escherichia coli: comparison of three phenotypic confirmation assays and genetic analysis. J Clin Microbiol. 2011;49(8):2924-32.

20. Tan TY, Ng LS, He J, Koh TH, Hsu LY. Evaluation of screening methods to detect plasmid-mediated AmpC in Escherichia coli, Klebsiella pneumoniae, and Proteus mirabilis. Antimicrob Agents Chemother. 2009;53(1):146-9.

21. Yum JH, Kim S, Lee H, Yong D, Lee K, Cho SN, et al. Emergence and wide dissemination of CTX-M-type ESBLs, and CMY-2- and DHA1-type AmpC beta-lactamases in Korean respiratory isolates of Klebsiella pneumoniae. J Korean Med Sci. 2005;20(6):961-5.

22. Mirzaee M, Pourmand MR, Chitsaz M, Mansouri S. Antibiotic resistance to third generation cephalosporins due to CTX-Mtype extended-spectrum $\beta$-lactamases isolates of Escherichia coli. Iran JPublic Health. 2009;38(1):10-17.

23. Ramazanzadeh R, Chitsaz M, Bahmani N. Prevalence and antimicrobial susceptibility of extended-spectrum beta-lactamaseproducing bacteria in intensive care units of Sanandaj general hospitals (Kurdistan, Iran). Chemotherapy. 2009;55(4):287-92.

24. Brigante G, Luzzaro F, Perilli M, Lombardi G, Coli A, Rossolini GM, et al. Evolution of CTX-M-type beta-lactamases in isolates of Escherichia coli infecting hospital and community patients. Int $J$ Antimicrob Agents. 2005;25(2):157-62.

25. Galas M, Decousser JW, Breton N, Godard T, Allouch PY, Pina P, et al. Nationwide study of the prevalence, characteristics, and molecular epidemiology of extended-spectrum-beta-lactamaseproducing Enterobacteriaceae in France. Antimicrob Agents Chemother. 2008;52(2):786-9.

26. Heffernan HM, Woodhouse RE, Pope CE, Blackmore TK. Prevalence and types of extended-spectrum beta-lactamases among urinary Escherichia coli and Klebsiella spp. in New Zealand. Int JAntimicrob Agents. 2009;34(6):544-9.

27. Mohamed Al-Agamy MH, El-Din Ashour MS, Wiegand I. First description of CTX-M beta-lactamase-producing clinical Escherichia coli isolates from Egypt. Int J Antimicrob Agents. 2006;27(6):545- 